\title{
Models to Assess the Effects of Nonsmooth Control and Stochastic Perturbation on Pest Control: A Pest-Natural-Enemy Ecosystem
}

\author{
Xuewen Tan, ${ }^{1,2}$ Wenjie Qin $\left(\mathbb{D},{ }^{3,4}\right.$ Guangyao Tang, ${ }^{5}$ Changcheng Xiang $\mathbb{D}^{5},{ }^{5}$ and Xinzhi Liu ${ }^{6}$ \\ ${ }^{1}$ School of Mathematics and Computer Science, Yunnan Minzu University, Kunming 650500, China \\ ${ }^{2}$ College of Mathematics and Information Science, Shaanxi Normal University, Xi'an 710062, China \\ ${ }^{3}$ Three Gorges Mathematical Research Center, China Three Gorges University, Yichang 443002, China \\ ${ }^{4}$ Laboratory for Industrial and Applied Mathematics, York University, Toronto M3J 1P3, Canada \\ ${ }^{5}$ Department of Mathematics, Hubei Minzu University, Enshi 445000, China \\ ${ }^{6}$ Department of Applied Mathematics, University of Waterloo, Waterloo N2L 3G1, Canada
}

Correspondence should be addressed to Wenjie Qin; wenjieqin@hotmail.com

Received 15 January 2019; Accepted 5 March 2019; Published 14 April 2019

Academic Editor: Xiaopeng Zhao

Copyright (C) 2019 Xuewen Tan et al. This is an open access article distributed under the Creative Commons Attribution License, which permits unrestricted use, distribution, and reproduction in any medium, provided the original work is properly cited.

\begin{abstract}
This paper investigates the impact of the threshold control strategy and environmental randomness on pest control. Firstly, a fixedtime impulsive stochastic ecosystem with IPM strategy is proposed, where the local and global existence of positive solution and the boundedness of expectation are discussed in detail. Moreover a sufficient condition for the extinction of the pest population with probability-1 is given. Then, a state-dependent stochastic ecosystem with IPM strategy is proposed. By employing the numerical simulations, the effects of ambient noise intensity on pest-outbreak are discussed. The result shows that there is a close relationship among the frequency of pest-outbreak, ET, the environmental perturbation intensity, and control measures. This study helps us to understand the impact of random factors on pest-outbreak frequency by theoretical derivations and numerical simulations; the results have directive significance in the design of an optimal control strategy for the department of ecological agriculture.
\end{abstract}

\section{Introduction}

Pest prevention and control have been attaching great importance by the agricultural departments as well as the management departments due to the outbreak of disaster pests. In the early days, chemical pesticides have played important roles in the pest control since pesticides can quickly kill a significant portion of a pest population, and it is convenient to carry out. However, the abuse of pesticides can also inevitably bring some negative effects such as the pest resistance and environment pollution. Therefore, a lot of control measures including chemical, physical, and biological methods have been proposed through long-term practice; Integrated Pest Management (IPM) is a combination of the above three tactics, which aims at the maximization of economic benefits [1-5] with the combination of the dynamical interaction between pests and their natural enemies and the implement of the comprehensive control strategies, in the hope of keeping the pest population under Economic Injury Levels (EIL).

Mathematical models can assist in designing strategies to control pest-outbreak. The application of IPM strategies has proven to have timeliness and transient effects. Moreover it also has made a crucial difference in the pest control because of the dynamical interaction based on intra-specific cooperation. Recently, many scholars have suggested using impulsive differential equations to investigate the dynamics of pest control models [6-15].

However, the effects of external random noises such as fire, flood, earthquake, and drought on the population cannot be ignored. For example, when the population is small or the noise intensity is high, it is not scientific enough to use the deterministic model. Meanwhile, it is necessary to establish a stochastic dynamic system to study the reality better in 
ecosystem. There were many scholars such as Kolmogorov $[16,17]$ and Feller $[18,19]$ who had done pioneering works in stochastic dynamic system. Meanwhile, in 1951 Itô established a stochastic integration [20-22]. In 1953, Doob published his results [23] and hence built up the mathematical theoretical foundation of stochastic dynamic system. Recently, the theory of the stochastic differential equation has gained renewed interest and also been applied to other fields [24-33].

In fact, the growth rate, environmental capacity, competition factors, and other parameters of the ecosystem are all subject to external random factors such as drought, harvest, fire, earthquakes, floods, deforestation, and hunting. Therefore, stochastic perturbations are introduced in the nonsmooth systems especially for pest-natural-enemy ecosystem being a very practical significance of the topic. The main propose of this paper is to investigate the effects of environmental randomness on IPM strategies. In order to do this, a fixed-time stochastic impulsive ecosystem with IPM strategies and a state-dependent stochastic ecosystem are proposed. Some theoretical derivations, qualitative analysis, numerical simulations are given in order to make the comprehensive and systematic research on those models, which could be in the hope of providing some suggestions for pest control and ecological management. Some theoretical, numerical, and biological analysis are given to investigate how environmental randomness affects the pest-outbreaks.

The organization of the present paper is as follows. In the next section, a fixed-time stochastic pest-natural-enemy ecosystem with IPM strategies is proposed, where the local and global existence of positive solution and the boundedness of expectation are discussed in detail. A sufficient condition for the extinction of pest population with probability 1 is given. In Section 3, with the establishment of a statedependent stochastic predator-prey ecosystem with IPM strategies and the development of the numerical simulations and methods, the effects of ambient noise intensity on pestoutbreak are discussed. The paper ends with some interesting biological conclusions, which complement the theoretical findings.

\section{A Fixed-Time Impulsive Stochastic Ecosystem with IPM Strategy}

2.1. Model Formulation. In 2001, in order to investigate the predator-prey interaction when the prey exhibits group defense, Xiao and Ruan [34] proposed the following model:

$$
\begin{aligned}
& \frac{\mathrm{d} x(t)}{\mathrm{d} t}=r x(t)\left[1-\frac{x(t)}{K}\right]-\frac{x(t) y(t)}{a+x^{2}(t)}, \\
& \frac{\mathrm{d} y(t)}{\mathrm{d} t}=y(t)\left[\frac{\mu x(t)}{a+x^{2}(t)}-D\right],
\end{aligned}
$$

where $x(t)$ and $y(t)$ represent the densities of prey (pest) and predator (natural enemy) populations, respectively; $r$ denotes the intrinsic growth rate and $K$ represents the carrying capacity for $x ; \mu$ and $d$ are the conversion rate and death rate for $y$, respectively; the sigmoidal saturation function, especially (i.e., a simplified Monod-Haldane or Holling IV function [35]), $\varphi(x)=x /\left[a+x^{2}(t)\right]$ describes the group defence of $x$.

Taking into account the effect of environmental noise and IPM strategies in model (1), then we can obtain the following fixed-time impulsive stochastic model

$$
\begin{aligned}
\mathrm{d} x(t)= & {\left[r x(t)\left(1-\frac{x(t)}{K}\right)-\frac{x(t) y(t)}{a+x(t)^{2}}\right] \mathrm{d} t } \\
& +\alpha_{1} x(t) \mathrm{d} B_{1}(t), \\
\mathrm{d} y(t)= & {\left[\frac{\mu x(t) y(t)}{a+x(t)^{2}}-D y(t)\right] \mathrm{d} t+\alpha_{2} y(t) \mathrm{d} B_{2}(t), } \\
x\left(t^{+}\right)= & (1-\theta) x(t), \quad t \neq n T, \\
y\left(t^{+}\right)= & y(t)+\tau,
\end{aligned}
$$

Here $T$ is the pulse control period, $\theta$ is the killing rate for pest population, and $\tau$ is the release constant for natural-enemy; those are the IPM control parameters; $\alpha_{i} \mathrm{~d} B_{i}(t)$, especially, denotes the white noise (i.e., an error term), $\alpha_{i}^{2}$ is a continuous bounded function on $R_{+}$representing the intensity of the noise, $B_{i}(t)$ is a Brownian motion defined on a complete probability space $(\Omega, \mathscr{F}, \mathscr{P})$, and $i=1,2$.

2.2. Preliminaries. For convenience, we will introduce some notations, definitions, and lemmas for impulsive stochastic differential equations (ISDE) [22, 24], which will be used for establishing our main results later.

Definition 1 (see [24]). For the impulsive stochastic differential equations,

$$
\begin{aligned}
& \mathrm{d} X(t)=f(t, X(t)) \mathrm{d} X t+\sigma(t, X(t)) \mathrm{d} X B(t), \\
& \quad t \neq n T \\
& X\left(t^{+}\right)=\left(1-p_{n}\right) X(t), \quad t=n T
\end{aligned}
$$

with initial condition $X\left(0^{+}\right)$. A stochastic process $X(t)=$ $\left(X_{1}(t), X_{2}(t), \cdots, X_{n}(t)\right)^{\tau}, t \in R_{+}$, is said to be a solution of model (3) if

(i) $X(t) \in C(0, T)$ is $\mathscr{F}_{t}$-adapted and each interval $(n T,(n+1) T) \quad \subset \quad R_{+}, n \in \mathcal{N} ; f(t, X(t)) \quad \epsilon$ $\mathscr{L}^{1}\left(R_{+} ; R_{n}\right), \sigma(t, X(t)) \in \mathscr{L}^{2}\left(R_{+} ; R_{n}\right)$, where $\mathscr{L}^{n}\left(R_{+} ; R_{n}\right)$ is all $R^{n}$ valued measurable $\mathscr{F}_{t}, g(t) \int_{0}^{\zeta}|g(t)|^{n} d t<\infty$ a.s. for every $\zeta>0$

(ii) There exists

$$
\begin{aligned}
& X\left(n T^{+}\right)=\lim _{t \longrightarrow n T^{+}} X(t), \\
& X\left(n T^{-}\right)=\lim _{t \longrightarrow n T^{-}} X(t), \\
& X\left(t_{n T}\right)=X\left(t_{n T}^{-}\right)
\end{aligned}
$$

with probability 1 for each $n T, n \in \mathcal{N}$; 
(iii) $X(t)$ obeys the integral equation

$$
\begin{aligned}
X(t)= & X(0)+\int_{0}^{t} f(s, X(s)) \mathrm{d} s \\
& +\int_{0}^{t} \sigma(s, X(s)) \mathrm{d} B(s)
\end{aligned}
$$

for almost all $t \in(0, T]$. And $X(t)$ obeys the integral equation

$$
\begin{aligned}
X(t)= & X\left(n T^{+}\right)+\int_{n T}^{t} f(s, X(s)) \mathrm{d} s \\
& +\int_{n T}^{t} \sigma(s, X(s)) \mathrm{d} B(s)
\end{aligned}
$$

for almost all $t \in(n T,(n+1) T], n \in \mathcal{N}$. Moreover, $X(t)$ satisfies the impulsive conditions at each $t=n T(n \in \mathcal{N})$ with probability 1.

Definition 2 (see [22]). If the pest population $x(t)$ satisfies $\lim _{t \rightarrow \infty} x(t)=0$, then $x(t)$ is said to be extinction with probability 1 .

Lemma 3 (the strong law of large numbers for martingales [22]). Let $x=\left\{x_{t}\right\}_{t \geq 0}$ be a local continuous martingale with $x_{0}=0$. If

$$
\lim _{t \rightarrow \infty} \sup \frac{\langle x, x\rangle_{t}}{t}<\infty \quad \text { a.s. }
$$

holds true, then

$$
\lim _{t \longrightarrow \infty} \frac{x_{t}}{t}=0 \quad \text { a.s. }
$$

Lemma 4 (Itô's formula [22]). Let $x(t, \omega)$ be defined in $t \geq 0$, and $x(t)$ satisfies

$$
\mathrm{d} x(t)=g(t) \mathrm{d} t+f(t) \mathrm{d} B(t),
$$

which is a d-Itô process, and $g \in \mathscr{L}^{1}\left(R_{+} ; R^{d}\right), f \in$ $\mathscr{L}^{2}\left(R_{+} ; R^{d \times m}\right)$. If $V(x, t) \in C^{2,1}\left(R^{d} \times R^{+} ; R\right)$, then $V(x(t), t)$ is Itô's with

$$
\begin{aligned}
& \mathrm{d} V(x(t), t)=\left[V_{t}(x(t), t)+V_{x}(x(t), t) g(t)\right. \\
& \left.\quad+0.5 \operatorname{Tr}\left(f^{T}(t) V_{x x}(x(t), t)\right) f(t)\right] \mathrm{d} t \\
& \left.\quad+V_{x x}(x(t), t)\right) f(t) \mathrm{d} B(t) \quad \text { a.s. }
\end{aligned}
$$

The comparison theorem of stochastic differential equation is one of the most important technologies to investigate the stochastic mathematical biology models; now it is given as follows.

Lemma 5 (comparison theorem of SDE [22]). Assume that $x_{i}(t), i=1,2$ is the solution for stochastic differential equation

$$
\mathrm{d} x_{i}(t)=f_{i}\left(x_{i}(t), t\right)+g\left(x_{i}(t), t\right) \mathrm{d} B_{i}(t),
$$

where $f(x, t) \in C([0, \infty) \times R), g(x, t) \in C([0, \infty) \times R)$ if (i) there exists a function $\rho(s)$ such that

$$
|g(t, x)-g(t, y)| \leq \rho(|x-y|), \quad x, y \in R, t \geq 0 .
$$

Meanwhile, $\rho(s)$ satisfies $\rho(0)=0$ and $\int_{0^{+}}^{+\infty} \rho(s) r m d s=$ $\infty, s \in[0, \infty)$;

(ii) $f_{1}(t, x)<f_{2}(t, x), x \in R, t \geq 0$;

(iii) $x_{1}(0) \leq x_{2}(0)$.

Then there exists $x_{1}(t) \leq x_{2}(t)$ with probability 1 for $t \geq 0$.

2.3. Mathematical Analysis for Model (2). In this section, the local and global existence of positive solution and the boundedness of expectation are discussed in detail, and the sufficient condition for the extinction of pest population with probability 1 is given. Firstly, the local existence of positive solution is given as follows.

Theorem 6. Model (2) exists a unique local positive solution $(x(t), y(t)), t \in\left[0, t^{*}\right)$ for each $\left(x_{0}^{+}, y_{0}^{+}\right) \in R_{+}^{2}, t^{*}$ being a finite number.

Proof. Consider the following stochastic differential equation

$$
\begin{aligned}
& \mathrm{d} x_{1}(t)=\left[r x_{1}(t)\left(1-\frac{(1-\theta) x_{1}(t)}{K}\right)\right. \\
& \left.-\frac{x_{1}(t)}{a+(1-\theta)^{2} x_{1}(t)^{2}}\left(y_{1}(t)+\tau\right)\right] \mathrm{d} t \\
& +\alpha_{1} x_{1}(t) \mathrm{d} B_{1}(t) \\
& \mathrm{d} y_{1}(t)=\left[\frac{\mu(1-\theta) x_{1}(t)\left(y_{1}(t)+\tau\right)}{a+(1-\theta)^{2} x_{1}(t)^{2}}\right. \\
& \left.-D\left(y_{1}(t)+\tau\right)\right] \mathrm{d} t+\alpha_{2}\left(y_{1}(t)+\tau\right) \mathrm{d} B_{2}(t)
\end{aligned}
$$

with $\left(x_{10}, y_{10}\right)=\left(x_{0}^{+}, y_{0}^{+}\right)$. Motivated by the main result of [27], (13) provides a unique local positive solution. Meanwhile, assume that

$$
\begin{aligned}
& x(t)=(1-\theta) x_{1}(t) \\
& y(t)=y_{1}(t)+\tau
\end{aligned}
$$

with $\left(x_{10}, y_{10}\right)=\left(x_{0}^{+}, y_{0}^{+}\right)$. Since $x(t)$ and $y(t)$ are continuous on $(0, T]$ and $t \in(n T,(n+1) T] \subset[0, \infty), n \in \mathcal{N}$. For any $x(t)$, it gives that

$$
\begin{aligned}
& \mathrm{d} x(t)=\mathrm{d}\left[(1-\theta) x_{1}(t)\right]=(1-\theta) \mathrm{d} x_{1}(t)=(1-\theta) \\
& \cdot\left[r x_{1}(t)\left(1-\frac{(1-\theta) x_{1}(t)}{K}\right)\right. \\
& \left.-\frac{x_{1}(t)}{a+(1-\theta)^{2} x_{1}(t)^{2}}\left(y_{1}(t)+\tau\right)\right] \mathrm{d} t+(1-\theta) \\
& \cdot \alpha_{1} x_{1}(t) \mathrm{d} B_{1}(t)=\left[r x(t)\left(1-\frac{x(t)}{K}\right)\right. \\
& \left.-\frac{x(t) y(t)}{a+x(t)^{2}}\right] \mathrm{d} t+\alpha_{1} x(t) \mathrm{d} B_{1}(t)
\end{aligned}
$$


and

$$
\begin{aligned}
x\left(n T^{+}\right) & =\lim _{t \longrightarrow n T^{+}} x(t)=\lim _{t \longrightarrow n T^{+}}(1-\theta) x_{1}(t) \\
& =\lim _{t \longrightarrow n T^{+}}(1-\theta) x_{1}\left(n T^{+}\right) \\
& =(1-\theta)(1-\theta) x_{1}(n T)=(1-\theta) x(n T)
\end{aligned}
$$

for every $n \in \mathcal{N}$ and $t \neq n T$. Furthermore, it is easy to derive

$$
\begin{aligned}
x\left(n T^{-}\right) & =\lim _{t \rightarrow n T^{-}} x(t)=\lim _{t \longrightarrow n T^{-}}(1-\theta) x_{1}(t) \\
& =\lim _{t \rightarrow n T^{-}}(1-\theta) x_{1}\left(n T^{-}\right)=(1-\theta) x_{1}(n T) \\
& =x(n T) .
\end{aligned}
$$

Analogously, for any $y(t)$, we can derive that

$$
\begin{aligned}
\mathrm{d} y(t) & =\mathrm{d}\left[y_{1}(t)+\tau\right]=\mathrm{d} y_{1}(t) \\
= & {\left[\frac{\mu(1-\theta) x_{1}(t)\left[y_{1}(t)+\tau\right]}{a+(1-\theta)^{2} x_{1}(t)^{2}}-D\left[y_{1}(t)+\tau\right]\right] \mathrm{d} t } \\
& +\alpha_{2}\left[y_{1}(t)+\tau\right] \mathrm{d} B_{2}(t) \\
= & {\left[\frac{\mu x(t) y(t)}{a+x(t)^{2}}-D y(t)\right] \mathrm{d} t+\alpha_{2} y(t) \mathrm{d} B_{2}(t) }
\end{aligned}
$$

and

$$
\begin{aligned}
y\left(n T^{+}\right) & =\lim _{t \longrightarrow n T^{+}} y(t)=\lim _{t \longrightarrow n T^{+}}\left(y_{1}(t)+\tau\right) \\
& =\tau+y_{1}\left(n T^{+}\right)=\tau+\tau+y_{1}(n T) \\
& =y(n T)+\tau
\end{aligned}
$$

for every $n \in \mathcal{N}$ and $t \neq n T$. Meanwhile, it gives

$$
\begin{aligned}
y\left(n T^{-}\right) & =\lim _{t \longrightarrow n T^{-}} y(t)=\lim _{t \longrightarrow n T^{-}}\left(y_{1}(t)+\tau\right) \\
& =y_{1}\left(n T^{-}\right)+\tau=\tau+y_{1}(n T)=y(n T) .
\end{aligned}
$$

According to Definition 1, model (2) provides a unique local positive solution. The proof of Theorem 6 is completed.

The existence and uniqueness of the local positive solution of model (2) are derived by Theorem 6, and some numerical simulations are also given, as shown in Figures 1 and 2. In particular, there are two cases representing the different noise density in Figures 1 and 2, which indicate the smaller the environmental disturbance is, the more effective the impulsive strategy is. Meanwhile, if the strong disturbance can cause the dynamical behaviors of system becoming more complex, it will bring some great challenges for pest control.

Next, we continue to study the global existence of positive solution for model (2).

Theorem 7. Model (2) provides a unique global positive solution $\left(x^{*}(t), y^{*}(t)\right)$ for any initial condition $\left(x_{0}^{+}, y_{0}^{+}\right) \in R_{+}^{2}$; it will remain in $R_{+}^{2}=\{(x, y) \mid x>0, y>0\}$ with probability 1 .

Proof. By applying Theorem 6, any solution of model (2) with $\left(x_{0}^{+}, y_{0}^{+}\right) \in R_{+}^{2}$ is a local positive solution. It follows from the first equation of (2) that

$$
\mathrm{d} x(t) \leq\left[r x(t)\left(1-\frac{x(t)}{K}\right)\right] \mathrm{d} t+\alpha_{1} x(t) \mathrm{d} B_{1}(t) .
$$

Consider the following comparison system:

$$
\begin{aligned}
& \mathrm{d} e(t)=r e(t)\left(1-\frac{e(t)}{K}\right) \mathrm{d} t+\alpha_{1} e(t) \mathrm{d} B_{1}(t), \\
& e\left(t^{+}\right)=(1-\theta) e(t), \quad t=n T, \\
& e\left(0^{+}\right)=x\left(0^{+}\right) .
\end{aligned}
$$

According to the main result of [24], model (22) provides a unique global positive solution

$$
e^{*}(t)=\frac{(1-\theta)^{n} \exp \left[\int_{0}^{t}\left(r-0.5 \alpha_{1}^{2}\right) \mathrm{d} s+\alpha_{1} \int_{0}^{t} \mathrm{~d} B(s)\right]}{1 / x_{0}+(1-\theta)^{n} \int_{0}^{t}\left\{(r / K) \exp \left[\int_{0}^{s}\left(r-0.5 \alpha_{1}^{2}\right) \mathrm{d} \tau+\alpha_{1} \int_{0}^{s} \mathrm{~d} B(\tau)\right]\right\} \mathrm{d} s}
$$

with $e\left(0^{+}\right)=x_{0}^{+}$, and it is continuous. By employing the comparison theorem of stochastic differential equation, we can obtain that

$$
0 \leq x(t) \leq e^{*}(t)
$$

Similarly, it follows from the second equation of model (2) that

$$
\mathrm{d} y(t) \leq\left[\mu e^{*}(t) y(t)-D y(t)\right] \mathrm{d} t+\alpha_{2} y(t) \mathrm{d} B_{2}(t),
$$

and then consider the following equation:

$$
\begin{aligned}
& \mathrm{d} g(t)=\left[\mu e^{*}(t) g(t)-D g(t)\right] \mathrm{d} t+\alpha_{2} g(t) \mathrm{d} B_{2}(t), \\
& t \neq n T, \\
& g\left(t^{+}\right)=g(t)+\tau, \quad t=n T, \\
& g\left(0^{+}\right)=y\left(0^{+}\right) .
\end{aligned}
$$




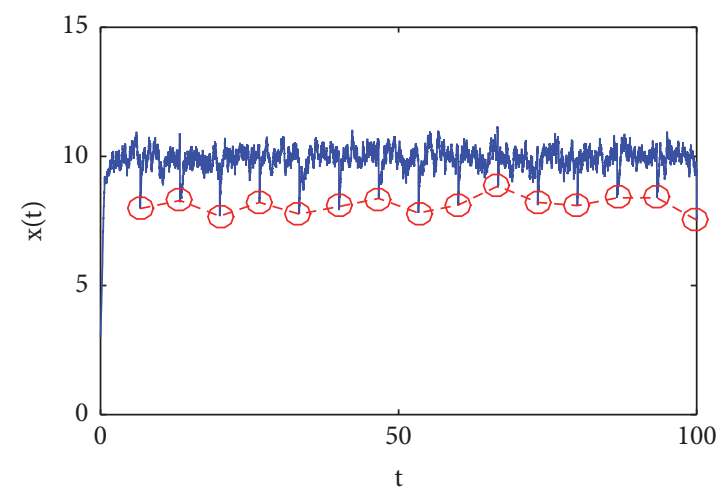

(a)

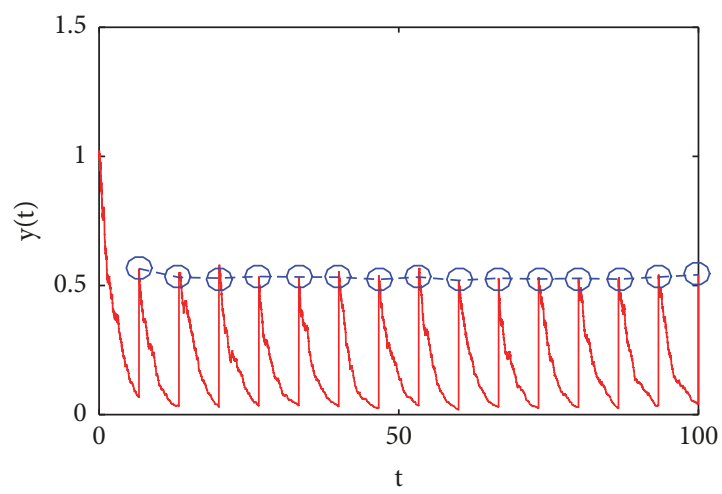

(b)

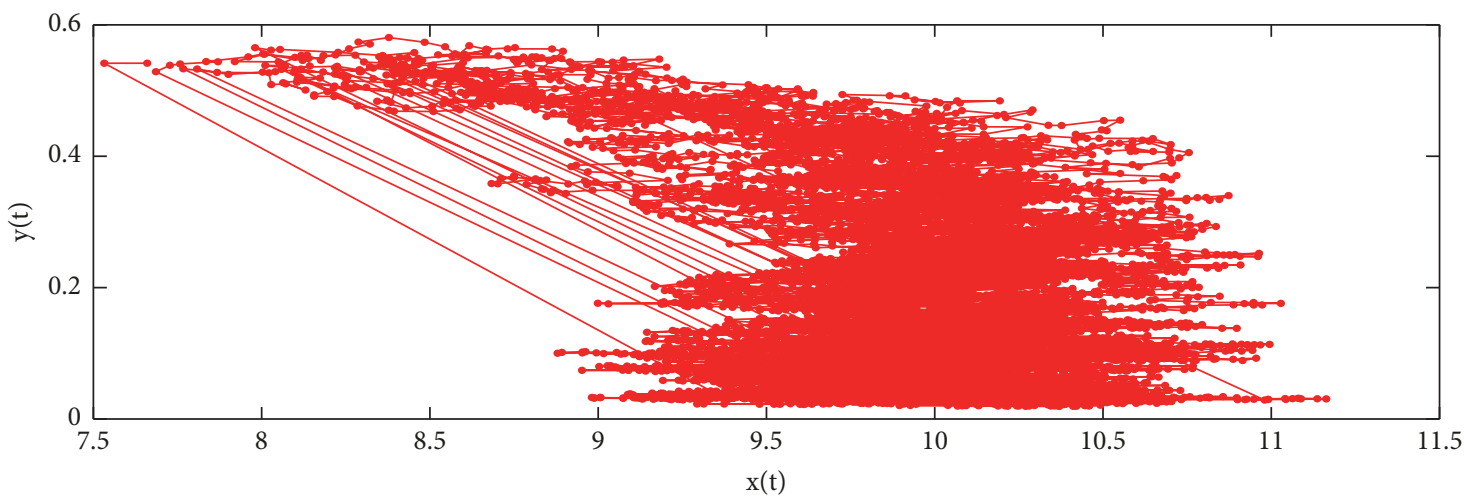

(c)

Figure 1: Solutions of model (2) with $\alpha_{1}=0.1, \alpha_{2}=0.15$. The other parameters are fixed as $r=2, K=5, a=1, D=0.5, \mu=0.8, \theta=0.2, \tau=$ $0.5, T=6.7,\left(x_{0}, y_{0}\right)=(3,1)$.

Obviously, the above equation (26) has a unique global positive solution

$$
\begin{aligned}
& g^{*}(t) \\
& \quad=g(0) \exp \left\{\int_{0}^{t}\left[\mu e^{*}(s)-D\right] \mathrm{d} s+\int_{0}^{t} \alpha_{2} \mathrm{~d} B_{2}(s)\right\} \\
& \quad+n \tau
\end{aligned}
$$

with $g\left(0^{+}\right)=y\left(0^{+}\right)>0$, and it is continuous.

Combining (24) and (27), model (2) provides a unique global positive solution $\left(x^{*}(t), y^{*}(t)\right)$ with initial value $x\left(0^{+}\right)>0, y\left(0^{+}\right)>0$. The proof of Theorem 7 is completed.

The above two theorems show that model (2) provides the local and global positive solution; we further investigate the extinction of the pest population.

Theorem 8. If the inequality $[\ln (1-\theta)] / T<0.5 \alpha_{1}^{2}-r$ holds true, then the pest population $x(t)$ of model (2) goes to extinction with probability 1 .

Proof. Use the transformation of the form

$$
x(t)=(1-\theta)^{n} \phi(t) .
$$

Applying Itô's formula to the first equation of model (2), it yields

$$
\begin{aligned}
\mathrm{d} \ln \phi(t) & =\frac{\mathrm{d} \phi(t)}{\phi(t)}-\frac{(\mathrm{d} \phi(t))^{2}}{2 \phi^{2}(t)} \\
& \leq\left[r-0.5 \alpha_{1}^{2}-\frac{(1-\theta)^{n} \phi(t)}{K}\right] \mathrm{d} t+\alpha_{1} \mathrm{~d} B(t) \\
& =\left[r-0.5 \alpha_{1}^{2}-\frac{x(t)}{K}\right] \mathrm{d} t+\alpha_{1} \mathrm{~d} B(t) .
\end{aligned}
$$

Integrating both sides of (29) from 0 to $t$, we can obtain that

$$
\begin{aligned}
\ln \phi(t)= & \ln \phi\left(0^{+}\right)+\int_{0}^{t}\left[r-0.5 \alpha_{1}^{2}-\frac{x(s)}{K}\right] \mathrm{d} s \\
& +M_{1}(t) .
\end{aligned}
$$

Assume that $M_{1}(t)=\alpha_{1} \int_{0}^{t} \mathrm{~d} B(s)$; then $M_{1}(t)$ is a local martingale whose quadratic variation is

$$
\left\langle M_{1}(t), M_{1}(t)\right\rangle=\alpha_{1}^{2} t .
$$

By the strong law of large numbers for martingales, we can derive that

$$
\lim _{t \rightarrow \infty} \frac{M_{1}(t)}{t}=0
$$




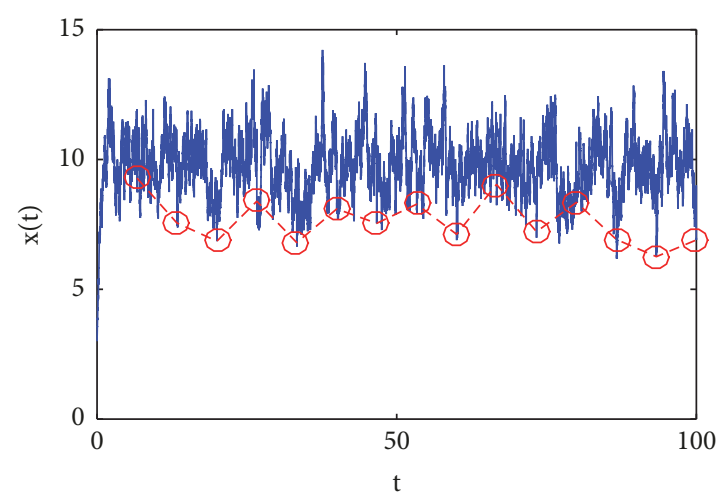

(a)

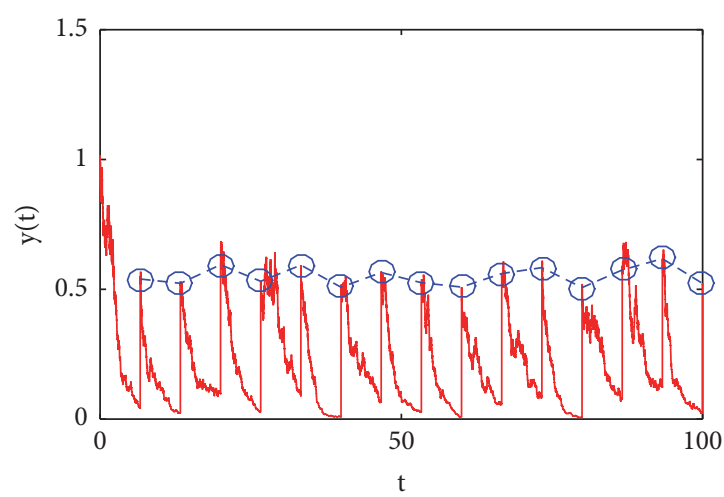

(b)

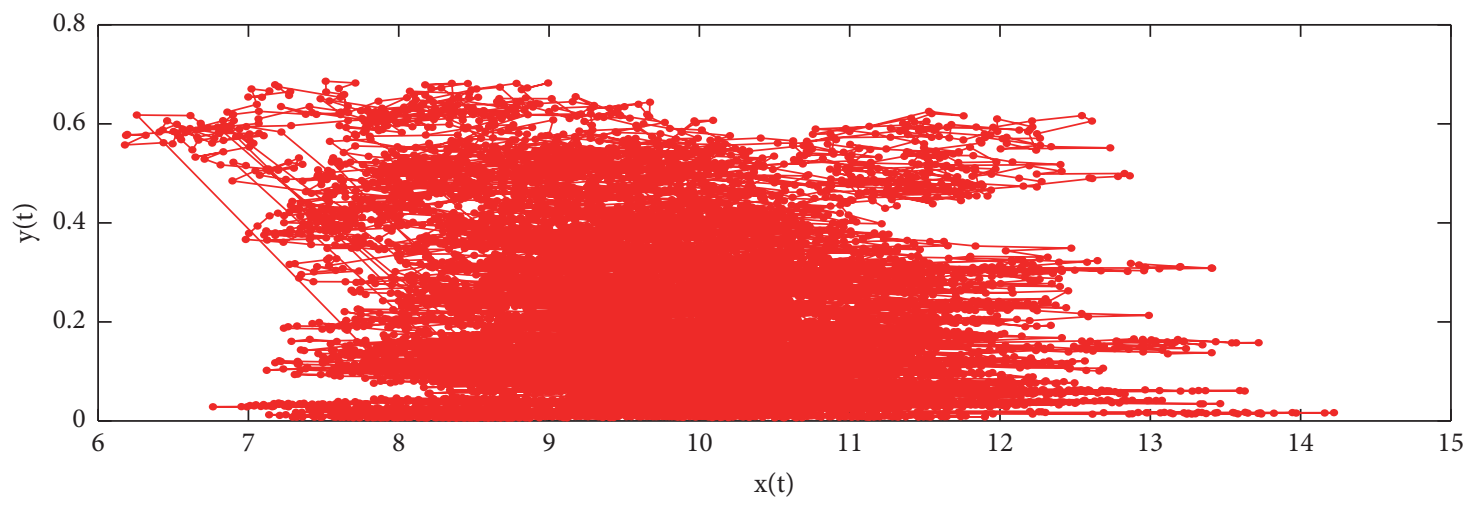

(c)

FIGURE 2: Solutions of model (2) with $\alpha_{1}=0.15, \alpha_{2}=0.25$. The other parameters are identified with Figure 1 .

Meanwhile, it follows from (30) that

$$
\begin{aligned}
n \ln (1-\theta)+\ln \phi(t)-\ln \phi\left(0^{+}\right) \\
=n \ln (1-\theta)+\int_{0}^{t}\left[r-0.5 \alpha_{1}^{2}-\frac{x(s)}{K}\right] \mathrm{d} s \\
\quad+M_{1}(t),
\end{aligned}
$$

that is,

$$
\begin{aligned}
\ln x(t)-\ln \phi\left(0^{+}\right)= & n \ln (1-\theta) \\
& +\int_{0}^{t}\left[r-0.5 \alpha_{1}^{2}-\frac{x(s)}{K}\right] \mathrm{d} s \\
& +M_{1}(t) .
\end{aligned}
$$

Therefore,

$$
\begin{aligned}
\ln x(t)-\ln \phi\left(0^{+}\right) \leq & n \ln (1-\theta)+\int_{0}^{t}\left[r-0.5 \alpha_{1}^{2}\right] \mathrm{d} s \\
& +M_{1}(t) .
\end{aligned}
$$

Combining $\lim _{t \rightarrow \infty} x(t)=0$ with Definition 1 , the pest population $x(t)$ of model (2) goes to extinction with probability 1. The proof of Theorem 8 is completed.

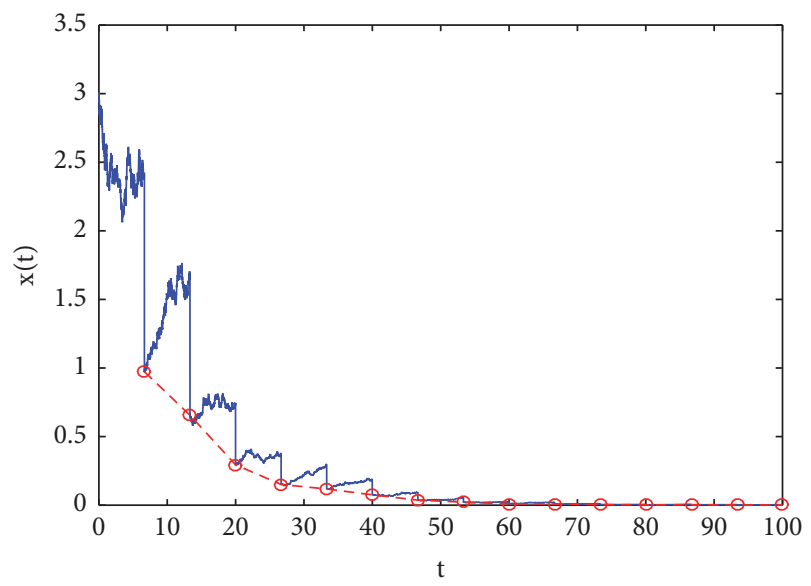

FIgURE 3: The pest-free solution of model (47). Parameters are fixed as $r=0.3, K=2, a=2, \mu=0.2, D=0.5, T=6.7, \theta=0.48, \tau=$ $0, \alpha_{1}=0.1, \alpha_{2}=0.15$.

In order to verify the main result of Theorem 8 , the numerical simulation is given as shown in Figure 3; it indicates that the pest population $x(t)$ goes to extinction when the IPM strategy including spraying pesticide with periodic is considered.

Furthermore, the mean boundedness of model (2) is discussed. 
Theorem 9. Assume that $(x(t), y(t))$ is a solution of model (2) with initial condition $\left(x\left(0^{+}\right), y\left(0^{+}\right)\right)$; then there exists a $L \in Z_{+}$ such that $E[x(t)] \leq L, E[y(t)] \leq L$; i.e., the solution of model (2) is mean boundedness.

Proof. Defining a function as $V(t)=\mu x(t)+y(t)$, and then applying the Itô's formula to solve the SDE about $V(t)$ along the solution of model (2)

$$
\begin{aligned}
\mathrm{d} V(t)= & \mu \mathrm{d} x(t)+\mathrm{d} y(t) \\
= & {\left[r \mu x(t)\left(1-\frac{x(t)}{K}\right)-\frac{\mu x(t) y(t)}{a+x(t)^{2}}\right] \mathrm{d} t } \\
& +\mu \alpha_{1} x(t) \mathrm{d} B_{1}(t) \\
& +\left[\frac{\mu x(t) y(t)}{a+x(t)^{2}}-D y(t)\right] \mathrm{d} t \\
& +\alpha_{2} y(t) \mathrm{d} B_{2}(t) .
\end{aligned}
$$

For each $t \in(n T,(n+1) T]$, integrating from $n T$ to $t$ on both sides of the above equation (36), it yields

$$
\begin{aligned}
V(t)= & V\left(n T^{+}\right) \\
& +\int_{n T}^{t}\left[r \mu x(s)\left(1-\frac{x(s)}{K}\right)-D y(s)\right] \mathrm{d} s \\
& +\int_{n T}^{t} \mu \alpha_{1} x(s) \mathrm{d} B_{1}(s)+\int_{n T}^{t} \alpha_{2} y(s) \mathrm{d} B_{2}(s) .
\end{aligned}
$$

Taking the mean value on both sides, we can obtain that

$$
\begin{aligned}
E[V(t)] & \\
=E & {\left[V\left(n T^{+}\right)\right] } \\
& +\int_{n T}^{t} E\left[r \mu x(s)\left(1-\frac{x(s)}{K}\right)-D y(s)\right] \mathrm{d} s,
\end{aligned}
$$

and it is easy to see that

$$
\begin{aligned}
\frac{\mathrm{d} E[V(t)]}{\mathrm{d} t}= & r \mu E\left[x(t)\left(1-\frac{x(t)}{K}\right)\right]-D E[y(t)] \\
= & (r u+\mu D) E[x(t)]-\frac{r \mu E\left[x^{2}(t)\right]}{K} \\
& -\mu D E[x(t)]-D E[y(t)] .
\end{aligned}
$$

Since

$$
\begin{aligned}
& \max \left\{(r u+\mu D) E[(t)]-\frac{r \mu E\left[x^{2}(t)\right]}{K}\right\} \\
& =\frac{(K+D) K}{4 r^{2} \mu},
\end{aligned}
$$

then

$$
\frac{\mathrm{d} E[V(t)]}{\mathrm{d} t} \leq \frac{(K+D) K}{4 r^{2} \mu}-D E[V(t)] .
$$

Meanwhile, according to model (2) we obtain

$$
\Delta E[V(t)]=\tau-\theta x(n T) \leq \tau .
$$

Combining (41) with (42), consider the following SDE

$$
\begin{aligned}
\mathrm{d} W(t) & =\left[\frac{(K+D) K}{4 r^{2} \mu}-D W(t)\right] \mathrm{d} t, \quad t \neq n T, \\
\Delta W(t) & =\tau, \quad t=n T .
\end{aligned}
$$

Obviously, it gives that

$W(t)$

$$
\begin{aligned}
= & \frac{(K+D) K}{4 r^{2} \mu D} \\
& +\left[W\left(0^{+}\right)-\frac{(K+D) K}{4 r^{2} \mu D}\right] \exp [-D(t-n T)], \\
& \quad t \in(n T,(n+1) T],
\end{aligned}
$$

where

$$
W\left(0^{+}\right)=\frac{(K+D) K}{4 r^{2} \mu D}+\frac{\tau}{[1-\exp (-D T)]} .
$$

By the comparison theorem of impulsive differential equation, it is easy to obtain

$$
\begin{aligned}
\lim _{t \rightarrow \infty} E[V(t)] & \leq \lim _{t \rightarrow \infty} E[W(t)] \\
& \leq \frac{(K+D) K}{4 r^{2} \mu D}+\frac{\tau}{[1-\exp (-D T)]} \triangleq L,
\end{aligned}
$$

and then the solution of model (2) is mean boundedness. The proof of Theorem 9 is completed.

\section{A State-Dependent Stochastic Ecosystem with IPM Strategy}

3.1. Model Formulation. In Section 2, a fixed-time stochastic impulsive ecosystem with IPM strategy has been investigated. The main purpose of this periodic control strategy is to eradicate pests rapidly and implement easily, and it is widely applied in agriculture and ecology. But at the same time it can bring serious environmental pollution and resource waste, so it is necessary to look for a new measure to control pest.

Taking the threshold control strategy into account means to maintain the density of the pests below the EIL rather than seeking to eradicate them. Therefore, in model (2), only the density of pests exceeds the given threshold, the combination of releasing the natural-enemy and spraying pesticide to 


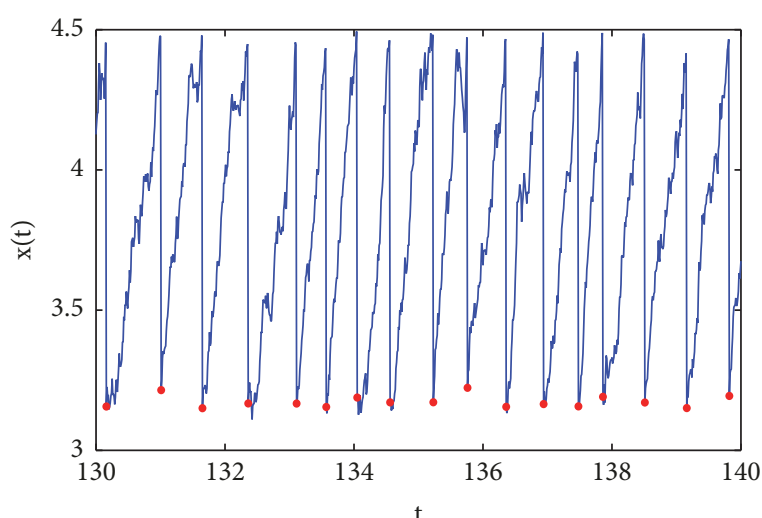

(a)

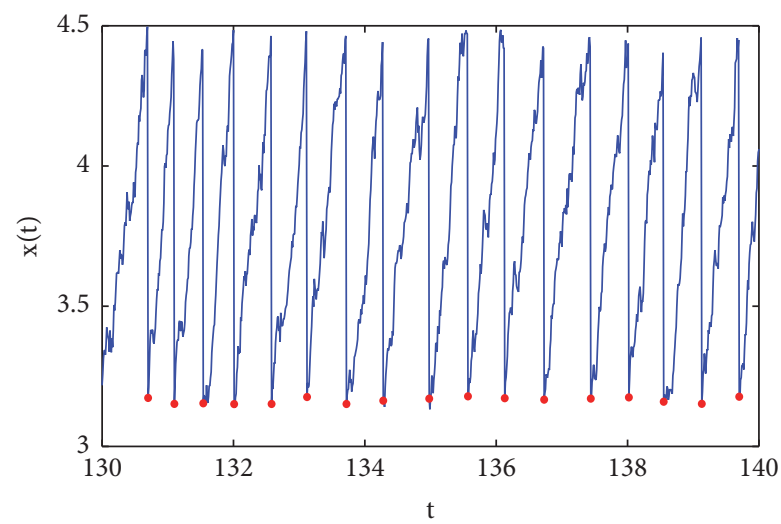

(c)

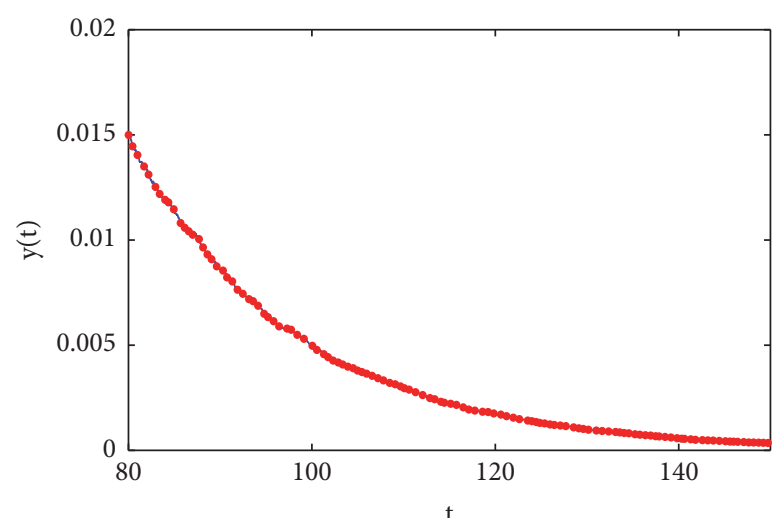

(b)

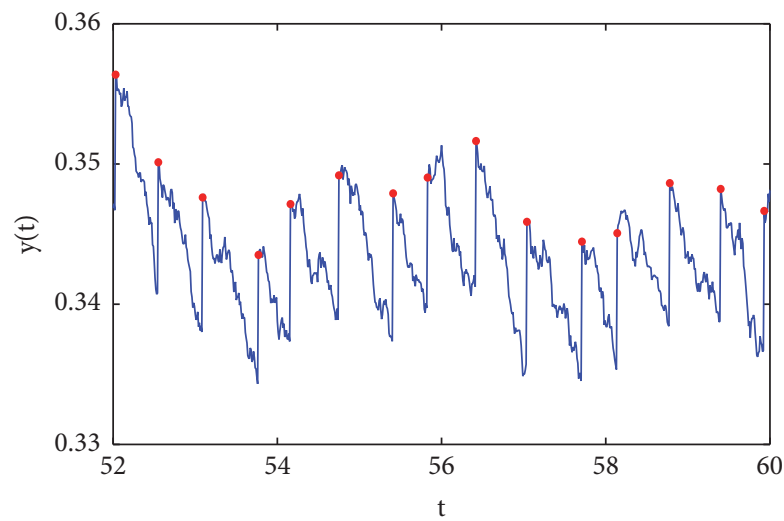

(d)

Figure 4: Two types of solutions of model (47). (a-b). Natural-enemy extinction solution with $\tau=0$; (c-d) Positive solution with $\tau=0.01$. The other parameters are fixed as $r=1, K=10, a=2, D=0.1, \mu=0.2, \theta=0.3, E T=4.5, \alpha_{1}=0.1, \alpha_{2}=0.015$.

suppress the pest under the level of ET. Motivated by this threshold control strategy, model (2) can be rewritten as

$$
\begin{array}{rlr}
\mathrm{d} x(t)= & {\left[r x(t)\left(1-\frac{x(t)}{K}\right)-\frac{x(t) y(t)}{a+x(t)^{2}}\right] \mathrm{d} t} & \\
& +\alpha_{1} x(t) \mathrm{d} B_{1}(t), \\
\mathrm{d} y(t)= & {\left[\frac{\mu x(t) y(t)}{a+x(t)^{2}}-D y(t)\right] \mathrm{d} t+\alpha_{2} y(t) \mathrm{d} B_{2}(t),} \\
x\left(t^{+}\right)= & (1-\theta) x(t), & x T, \\
y\left(t^{+}\right)= & y(t)+\tau, &
\end{array}
$$

Model (47) describes the threshold strategy and the environment random; this stochastic perturbation could take some serious challenge to pest control; we will investigate the effects of the stochastic perturbation on pest control or IPM strategy in the next subsection.

3.2. Numerical Simulations for Model (47). Comparing with model (2), model (47) describes the threshold control policy and stochastic perturbation more complexly; the term of environment random especially increases the uncertainty of the model (47), so it is difficult to investigate model (47) theoretically, so we can only investigate the dynamical behavior of model (47) by numerical simulations, and the analysis will focus on the pest-outbreak frequency and some key factors.

Firstly, the effect of releasing natural-enemy on dynamical behaviors of model is explored. On one hand, if there is no releasing natural-enemy (i.e., $\tau=0$ ), model (47) provides a boundary solution $(x(t), 0)$; in other words, the naturalenemy population $y$ finally goes to extinction, while the pest population $x$ goes to periodical vibration randomly. Although natural-enemy population is extinct, the pest population is still under control(i.e., $x \leq E T$ ), only to show the economic value with a high frequency, as shown in Figures 4(a) and 4(b). On the other hand, Figures 4(c) and 4(d) show that if there is releasing natural-enemy to ecosystem (i.e., $\tau=0.01$ ), both pest- and nature-enemy populations can coexist; two populations can be periodical vibration randomly. Comparing with Figure 4, the dynamical behavior of model (47) has a great change as shown in Figure 5. In particular, Figure 5 represents model (47) has several stages of pest-outbreak and relative stable. The curves of pestoutbreak include several rapid impulsive processes, while the 


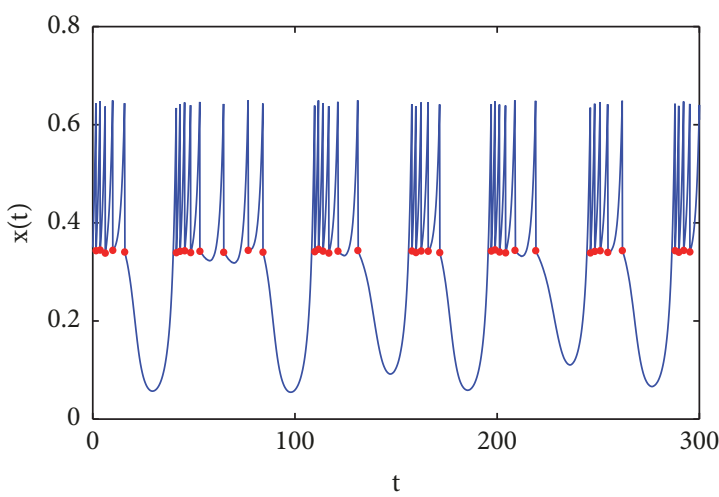

(a)

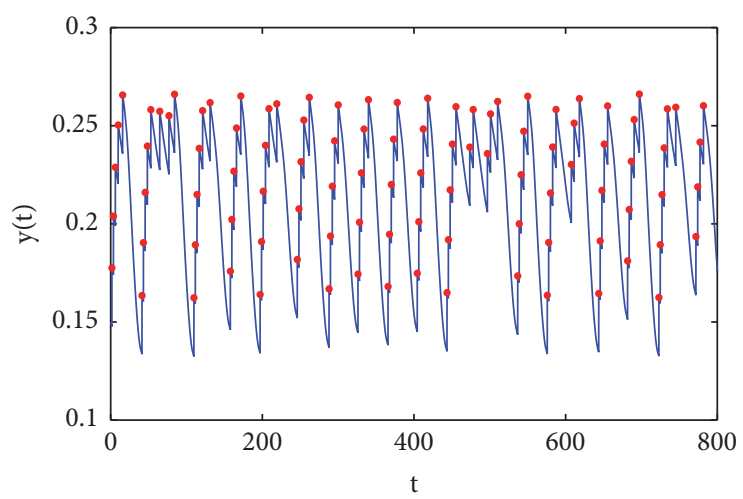

(b)

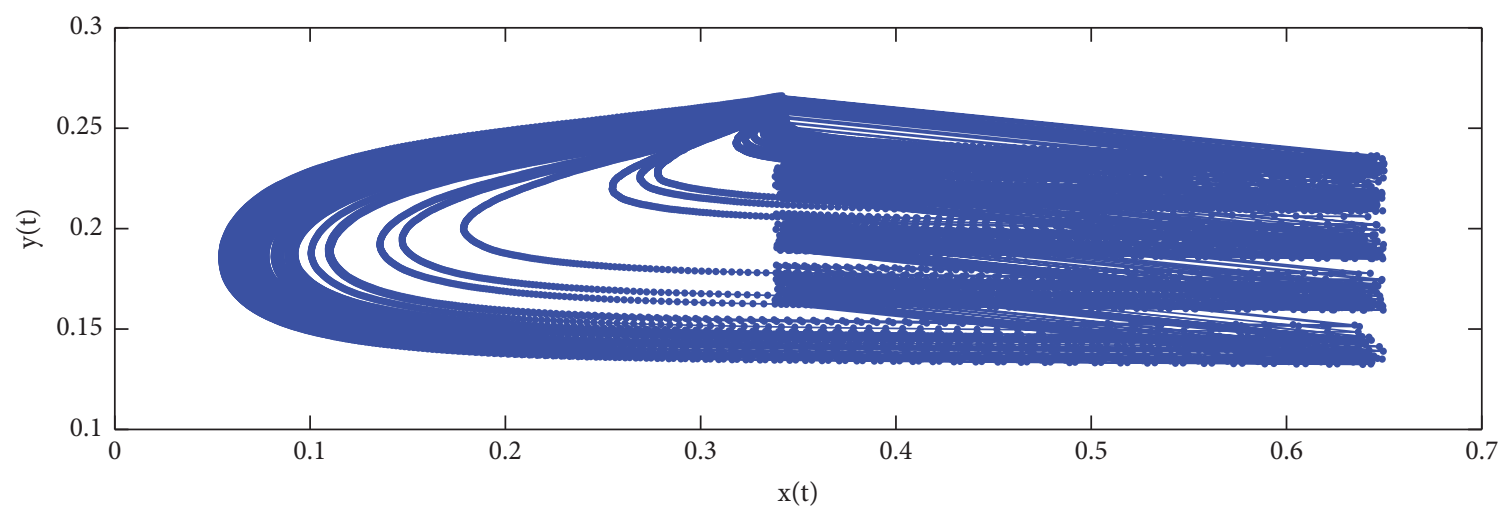

(c)

Figure 5: Solutions of model (47), parameters are fixed as $r=1, K=2, a=0.188, \mu=0.035, D=0.05, E T=0.65, \theta=0.48, \tau=0.03, \alpha_{1}=$ $0.01, \alpha_{2}=0.005$.

ones of pest stability have the length impulsive processes with two time intervals. In the initial stage, the density of natural-enemy keeps in relative levels, but the density of pest population increases rapidly. As long as releasing naturalenemy many times, pest will be relative stable. However, if the density of natural-enemy is in a low level, the outbreak of disaster pests will form again.

As we know, the state-dependent model has a switching time between $x<E T$ and $x<E T$; it could play a very important role on pest control. The shorter the switching time, the higher the pest-outbreak frequency; in other words, it is very frequent to spray pesticide and release naturalenemy, and it can cause serious environmental pollution and resource waste. It is difficult to design the proper measures if we do not know when and how to implement control strategies. Aimed at the state-dependent stochastic model (47), we further investigate the pest-outbreak frequency by applying the average switching time as the following method.

Step 1. For the given economic threshold ET in model (47), assume the times of pest arrivals at ET with $T=100$ are $N_{i}$, denoting the time interval between the first and $N_{i}$-th arrivals at ET is $\Delta T_{i}$, and then the average switching time is $\overline{T_{i}}=\left(\Delta T_{i}\right) /\left[N_{i}-1\right]$.
Step 2. Following Step 1, the average switching time is $T=\left(\sum_{i=1}^{N} \overline{T_{i}}\right) / N$ with $N=1000$. The effects of key parameters on the average pest-outbreak frequency are taken into account.

Now, we are in a position to investigate the effects of the economic threshold, the environmental perturbation intensity, and the IPM control measures on pest-outbreak frequency.

(i) The Effect of ET on Pest-Outbreak Frequency. Assume that ET is 4.5, 5.5, 6.5 and 7.5 in Figures 6(a), 6(b), 6(c), and 6(d) respectively. When $N=1000$, the frequency histograms are derived. According to $\overline{T_{i}}=\left[\Delta T_{i}\right] /\left[N_{i}-1\right]$, the pest-outbreak frequency is $0.9710,1.1354,1.2946,1.5105$, respectively. The result shows that there is a close relationship among the frequency of pest-outbreak, ET and the switching time. The smaller the value of ET is (or the shorter the switching time is), the higher the pest-outbreak frequency becomes.

(ii) The Effects of the Environmental Perturbation Intensity on Pest-Outbreak Frequency. According to choosing the variable perturbation intensity $\alpha_{1}$ and $\alpha_{2}$, the average time of pest population arrivals at ET is derived, and the frequency 


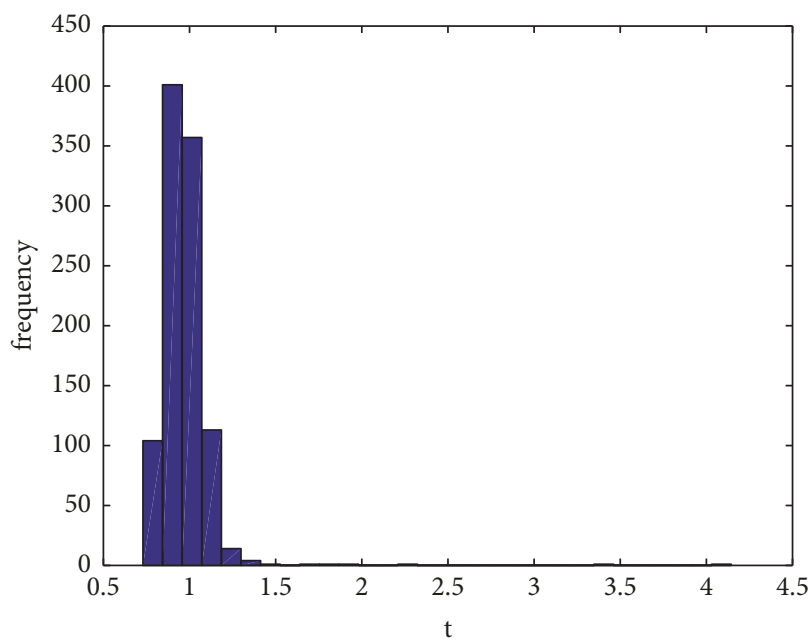

(a)

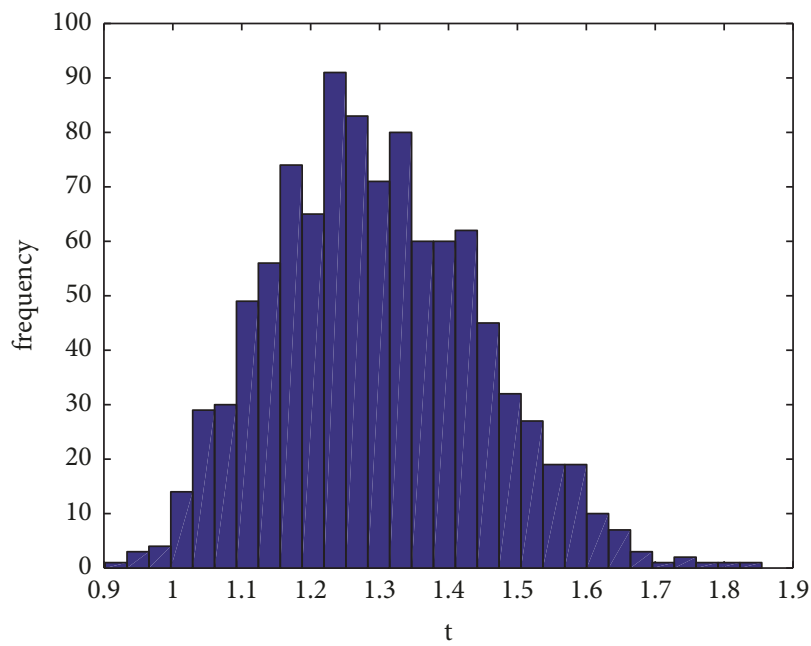

(c)

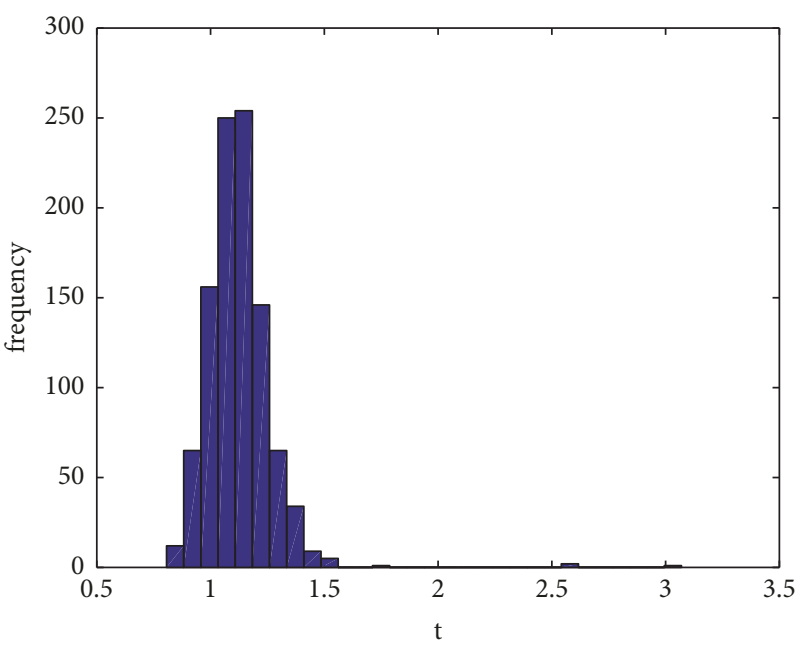

(b)

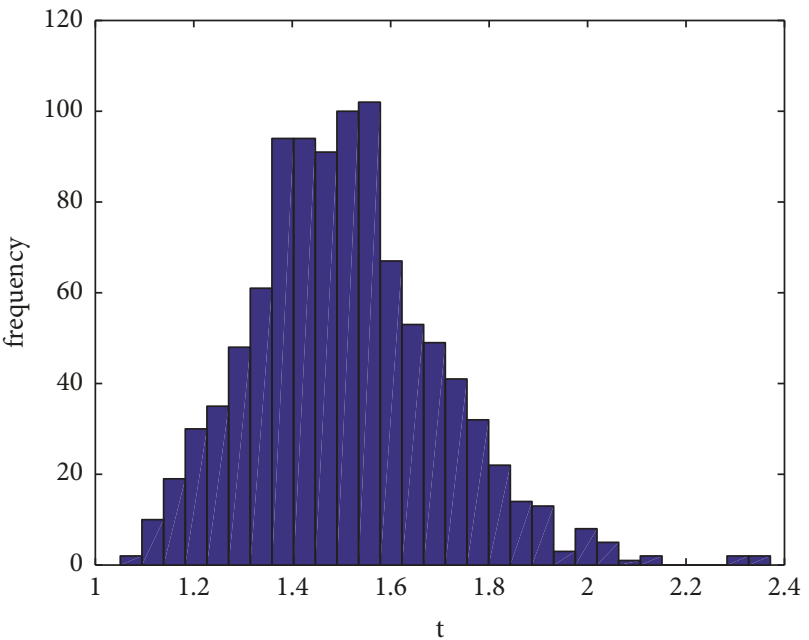

(d)

FigurE 6: The effects of ET on pest-outbreak frequency; parameters are fixed as $r=1, K=10, a=2, \mu=0.2, D=0.1, \theta=0.4, \tau=0, \alpha_{1}=$ $0.3, \alpha_{2}=0.25$, and (a) $E T=4.5$; (b) $E T=5.5$; (c) $E T=6.5$; (d) $E T=7.5$.

histogram is given, as shown in Figure 7. It is easy to see that the average time $\bar{T}$ is $1.4841,1.7936,1.5068,1.4178$ in the cases of Figures 7(a), 7(b), 7(c), and 7(d), respectively. The perturbation intensity has a great impact on pest population. It shows that the greater the impact of the perturbation intensity on pest population is, the lower the pest-outbreak frequency becomes. And the opposite is true for their natural enemies. The inescapable conclusion is that the stronger environmental perturbation intensity or a relative stable level of natural-enemy population could help the pest control. On the other hand, it is good for pest-outbreak.

(iii) The Effects of Control Measures on Pest-Outbreak Frequency. Spraying pesticide and releasing natural-enemy are the main measures of IPM strategies, so we vary the control parameters $\theta$ and $\tau$ to investigate the average time $\bar{T}$ and frequency histogram, as shown in Figure 8. As $n=1000$ times, the average time of pest population arrivals at ET is $0.42224,1.5043,1.7743,3.4562$, respectively, corresponding to each case in Figures 8(a), 8(b), 8(c), and 8(d). It indicates that the larger the value of $\theta$ and $\tau$ is, the lower the pestoutbreak frequency is. Compared with two control parameters, the pest-outbreak frequency could be most affected by $\tau$.

Based on the above analysis there is a close relationship among the frequency of pest-outbreak, ET, the environmental perturbation intensity, and control measures. The larger the value of ET is, the slower the pest-outbreak frequency becomes. Besides, the perturbation intensity also has a great impact on pest population. The greater the impact of the perturbation intensity on pest population is, the lower the pest-outbreak frequency becomes. And the opposite is true for their natural enemies. We can also reduce the pestoutbreak frequency by increasing the intensity of control measures. Finally, this study helps us to understand the 


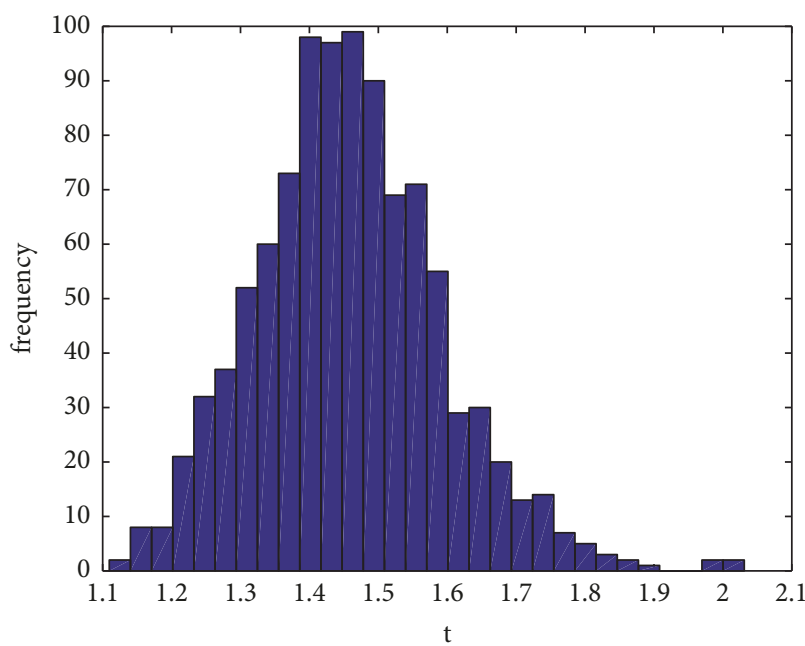

(a)

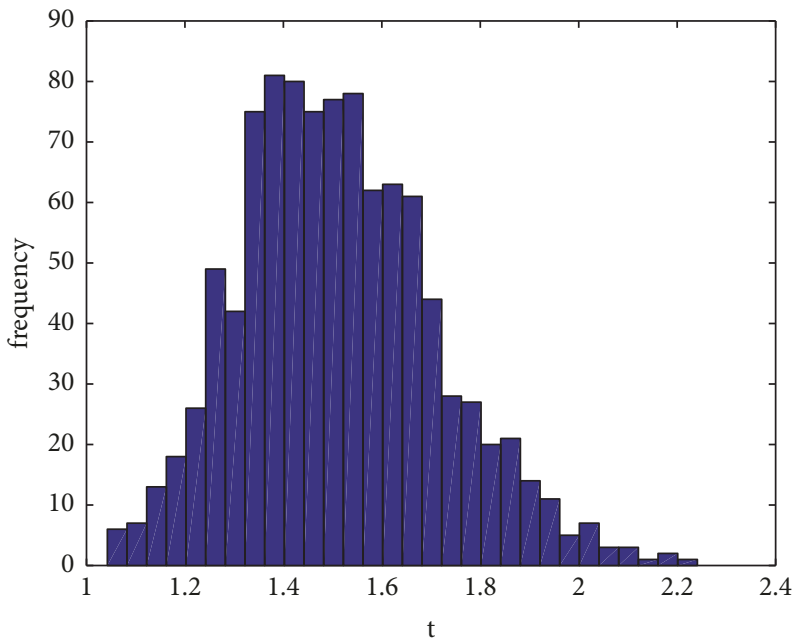

(c)

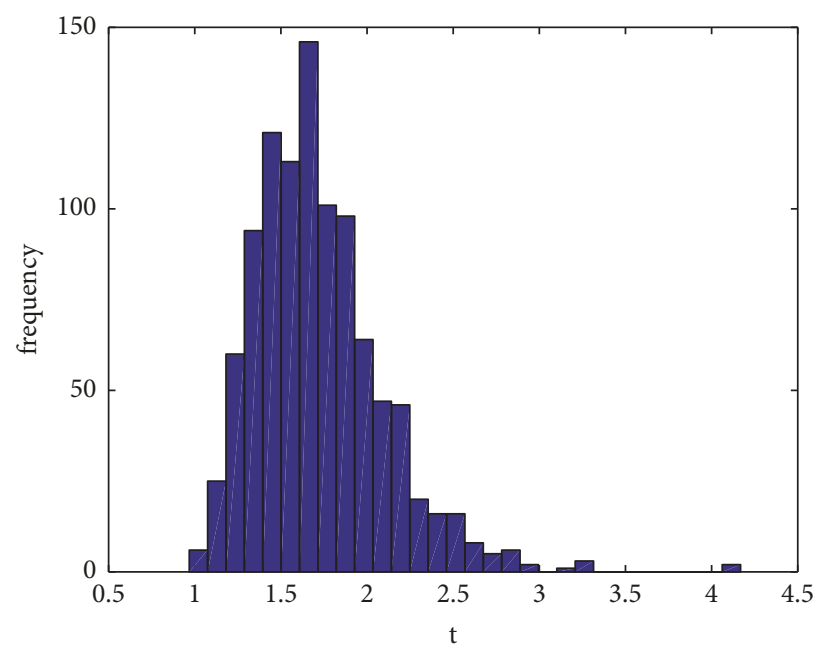

(b)

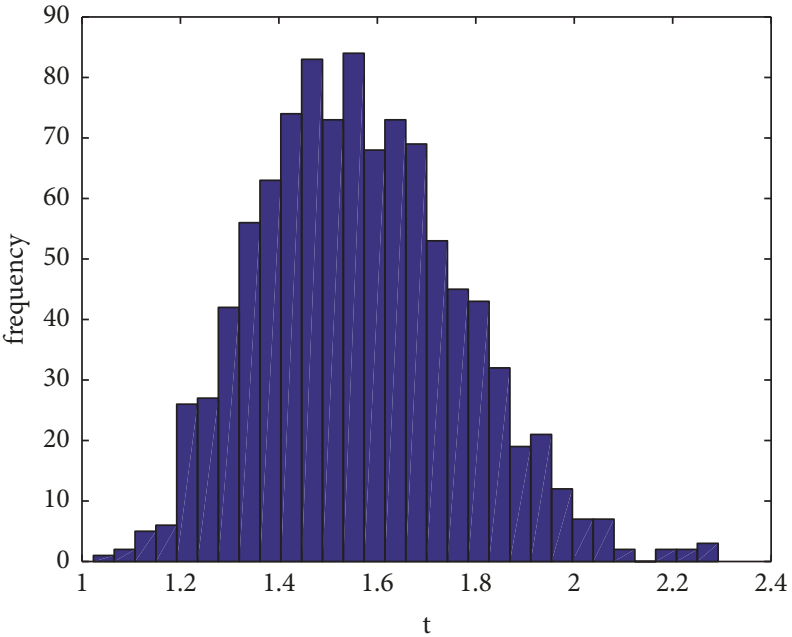

(d)

FIGURE 7: The effects of the environmental perturbation intensity on pest-outbreak frequency; parameters are fixed as $r=1, K=10, a=$ $2, \mu=0.2, D=0.1, E T=7.5, \theta=0.4, \tau=0$, and (a) $\alpha_{1}=0.2, \alpha_{2}=0.25$; (b) $\alpha_{1}=0.5, \alpha_{2}=0.25$; (c) $\alpha_{1}=0.3, \alpha_{2}=0.1 ;$ (d) $\alpha_{1}=0.3, \alpha_{2}=0.4$.

impact of random factors on pest-outbreak frequency by theoretical derivations and numerical simulations; the results have directive significance in the design of an optimal control strategy for the department of ecological agriculture.

\section{Conclusion and Discussion}

As we know pest-outbreaks often cause serious ecological and economic problems, requiring complex control strategies to reduce the loss because of the outbreak of disaster pests. Among the current control and preserve measures, the chemical control may be still one of the most important measures and can control plant diseases and insect pests effectively in a short time, but it will bring many serious problems including environment pollution and pesticide residual effects. Therefore, combining the chemical control, physical control, biological control, and some advanced information technologies, IPM strategy has established an efficient comprehensive control system to improve the efficiency of pest control.

Meanwhile, in order to describe the environmental randomness on pest control, this paper has proposed some impulsive stochastic dynamical models. For a fixed-time impulsive stochastic ecosystem, the local existence, global existence, and the mean boundedness of positive solutions of the system are investigated, and the sufficient conditions for pest-free solution with probability 1 are derived. For a statedependent stochastic ecosystem, the corresponding numerical simulations and methods are developed and the effects of ambient noise intensity on pest-outbreak are discussed. The result shows that there is a close relationship between the frequency of pest-outbreak, ET, the environmental perturbation intensity, and control measures.

The numerical results, especially, indicate that the larger the value of ET is, the slower the pest-outbreak frequency becomes. Besides, the perturbation intensity also has a great 


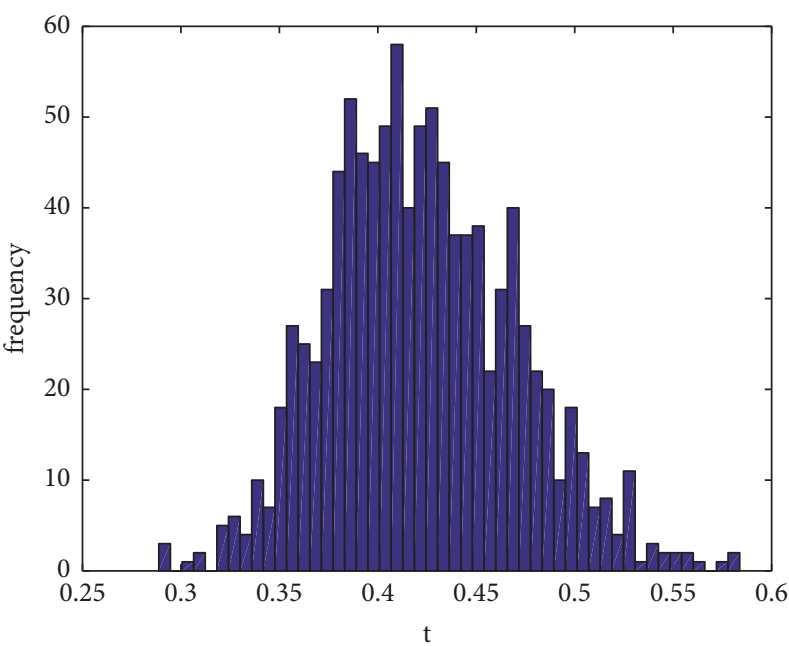

(a)

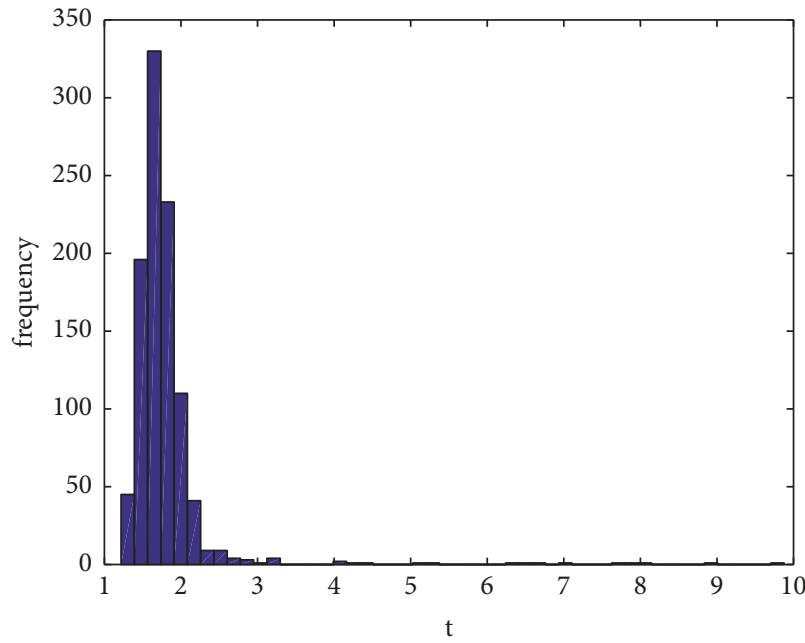

(c)

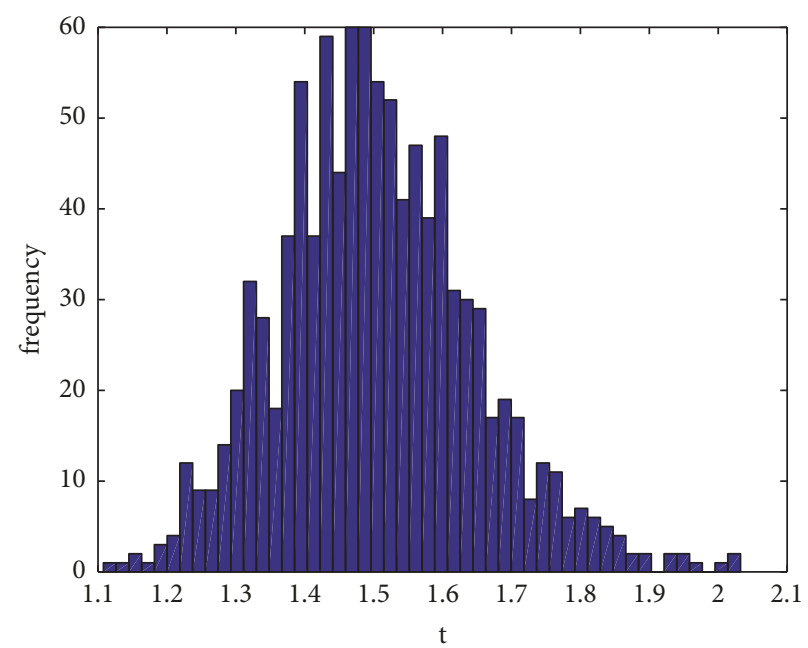

(b)

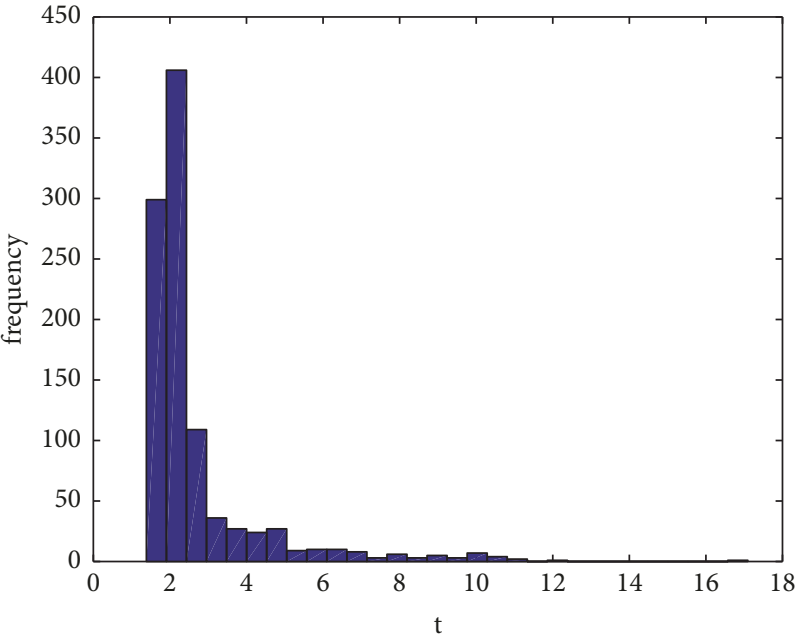

(d)

FIGURE 8: The effects of the control measures on pest-outbreak frequency; parameters are fixed as $r=1, K=10, a=2, \mu=0.2, D=0.1, E T=$ $7.5, \alpha_{1}=0.2, \alpha_{2}=0.25$, and (a) $\theta=0.1, \tau=0$; (b) $\theta=0.4, \tau=0$; (c) $\theta=0.4, \tau=0.2$; (d) $\theta=0.4, \tau=0.5$.

impact on pest population. The greater the impact of the perturbation intensity on pest population is, the lower the pest-outbreak frequency becomes. And the opposite is true for their natural enemies. We can also reduce the pestoutbreak frequency by increasing the intensity of control measures. Finally, this study helps us to understand the impact of random factors on pest-outbreak frequency by theoretical derivations and numerical simulations; the results have directive significance in the design of an optimal control strategy for the department of ecological agriculture.

However, the impulsive stochastic differential equation is a new research topic. Especially for the state-dependent stochastic dynamical model, it is first proposed in this paper, since the state-dependent stochastic model describes the threshold control policy and IPM strategy more complexly, that it is difficult to investigate this model theoretically, so we have investigated it by numerical simulations. Therefore, there are still many challenges in theoretical analysis and numerical research as well as biological conclusions, etc. It is necessary to develop new research methods to analyze statedependent impulsive stochastic models. We will continue to focus on the latest research results of those topics in later studies and conduct further and systematic research.

\section{Data Availability}

No data were used to support this study.

\section{Conflicts of Interest}

The authors declare that they have no competing interests.

\section{Authors' Contributions}

All the authors contributed equally and significantly to this paper. All the authors read and approved the final manuscript. Xuewen Tan (the first) wrote the body of the 
manuscript. Wenjie Qin (the second) provided the main ideas for the manuscript, biological explanations, and discussion. Guangyao Tang (the third) provided some important ideas for modelling. Changcheng Xiang (the fourth) provided the numerical simulations using MATLAB. Xinzhi Liu (The fifth) revised all the manuscript with structure, mathematics, and English writing.

\section{Acknowledgments}

We would like to thank Prof. Sanyi Tang of Shaanxi Normal University for helping us revise the manuscript that greatly improved the presentation of this paper. This work was supported by National Natural Science Foundation of China (no. 11601268, 11761031, 11601474) and China Scholarship Council to visit York University (no. 201808420192).

\section{References}

[1] M. L. Flint, Integrated Pest Management for Walnuts, California University of Integrated Pest Management Program. Division of Agriculture and Natural Resources, California, USA, 1987.

[2] J. C. Van Lenteren and J. Woets, "Biological and integrated pest control in greenhouses," Annual Review of Entomology, vol. 33, no. 1, pp. 239-250, 1988.

[3] J. C. Van Lenteren, "Integrated pest management in protected crops," in Integrated Pest Management: Principles And Systems Development, 1995.

[4] G. Gurr and S. Wratten, "Success in biological control of arthropods by augmentation of natural enemies," in Biological Control: Measures of Success, Springer, Dordrecht, Netherlands, 2000.

[5] S. N. Alam, M. I. Hossain, F. M. A Rouf et al., "Implementation and promotion of an IPM strategy for control of eggplant fruit and shoot borer in South Asia," AVRDC, vol. no. 36, 2006.

[6] D. Bainov and P. Simeonov, Impulsive differential equations: periodic solutions and applications, Longman Scientific \& Technical, London, UK, 1993.

[7] V. Lakshmikantham, D. D. Bainov, and P. S. Simeonov, Theory of Impulsive Differential Equations, World Scientific, Singapore, 1989.

[8] S. Tang and L. Chen, "Modelling and analysis of integrated pest management strategy," Discrete and Continuous Dynamical Systems - Series B, vol. 4, no. 3, pp. 759-768, 2004.

[9] B. Liu, L. S. Chen, and Y. J. Zhang, "The dynamics of a preydependent consumption model concerning impulsive control strategy," Applied Mathematics and Computation, vol. 169, no. 1, pp. 305-320, 2005.

[10] P. Georgescu, H. Zhang, and L. Chen, "Bifurcation of nontrivial periodic solutions for an impulsively controlled pest management model," Applied Mathematics and Computation, vol. 202, no. 2, pp. 675-687, 2008.

[11] S. Y. Tang, G. Y. Tang, and R. A. Cheke, "Optimum timing for integrated pest management: modelling rates of pesticide application and natural enemy releases," Journal of Theoretical Biology, vol. 264, no. 2, pp. 623-638, 2010.

[12] S. Tang, J. Liang, Y. Tan, and R. A. Cheke, "Threshold conditions for integrated pest management models with pesticides that have residual effects," Journal of Mathematical Biology, vol. 66, no. 1-2, pp. 1-35, 2013.
[13] S. Y. Tang, W. H. Pang, R. A. Cheke, and J. H. Wu, "Global dynamics of a state-dependent feedback control system," Advances in Difference Equations, vol. 2015, article 322, 2015.

[14] S. Tang, X. Tan, J. Yang, and J. Liang, "Periodic solution bifurcation and spiking dynamics of impacting predator-prey dynamical model," International Journal of Bifurcation and Chaos, vol. 28, no. 12, 1850147, 32 pages, 2018.

[15] P. Wang, W. Qin, and G. Tang, "Modelling and analysis of a hostparasitoid impulsive ecosystem under resource limitation," Complexity, vol. 2019, Article ID 9365293, 12 pages, 2019.

[16] A. N. Kolmogorov, "On the statistical theory of the crystallization of metals," Bulletin of the Academy of Sciences of the USSR, Mathematics Series, vol. 1, pp. 355-359, 1937.

[17] B. V. Gnedenko and A. N. Kolmogorov, Independent Random Variables, Addison-Wesley, Massachusetts, USA, 1954.

[18] W. Feller, "The asymptotic distribution of the range of sums of independent random variables," Annals of Mathematical Statistics, vol. 22, pp. 427-432, 1951.

[19] W. Feller, An Introduction to Probability Theory and ITS Applications, John Wiley \& Sons, New York, NY, USA, 1957.

[20] K. Ito, Memoirs of the American Mathematical Society, American Mathematical Society, Providence, RI, USA, 1951.

[21] K. Itô, "On a formula concerning stochastic differentials," Nagoya Mathematical Journal, vol. 3, pp. 55-65, 1951.

[22] X. R. Mao, Stochastic Differential Equations and Applications, Woodhead Publishing, Cambridge, UK, 2007.

[23] J. L. Doob, Stochastic Processes, John Wiley \& Sons, New York, NY, USA, 1953.

[24] M. Liu and K. Wang, "On a stochastic logistic equation with impulsive perturbations," Computers \& Mathematics with Applications, vol. 63, no. 5, pp. 871-886, 2012.

[25] X. Li, A. Gray, D. Jiang, and X. Mao, "Sufficient and necessary conditions of stochastic permanence and extinction for stochastic logistic populations under regime switching," Journal of Mathematical Analysis and Applications, vol. 376, no. 1, pp. 1128, 2011.

[26] R. Wu, X. Zou, and K. Wang, "Asymptotic properties of a stochastic Lotka-Volterra cooperative system with impulsive perturbations," Nonlinear Dynamics, vol. 77, no. 3, pp. 807-817, 2014.

[27] R. H. Wu, X. L. Zou, and K. Wang, "Asymptotic behavior of a stochastic non-autonomous predator-prey model with impulsive perturbations," Communications in Nonlinear Science and Numerical Simulation, vol. 20, no. 3, pp. 965-974, 2015.

[28] L. Wang, X. Wang, and Q. Zhang, "Permanence and extinction of a high-dimensional stochastic resource competition model with noise," Advances in Difference Equations, Paper No. 441, 18 pages, 2018.

[29] M. Obradovi, "Implicit numerical methods for neutral stochastic differential equations with unbounded delay and Markovian switching," Applied Mathematics and Computation, vol. 347, pp. 664-687, 2019.

[30] Y. Zhang, K. Fan, S. Gao, Y. Liu, and S. Chen, "Ergodic stationary distribution of a stochastic SIRS epidemic model incorporating media coverage and saturated incidence rate," Physica A: Statistical Mechanics and its Applications, vol. 514, pp. 671-685, 2019.

[31] X. R. Mao and C. G. Yuan, Stochastic Differential Equations with Markovian Switching, Imperial College Press, London, UK, 2006. 
[32] S. Hu, Stochastic Differential Equations, Science Press, Beijing, China, 2007.

[33] S. Zhang, X. Meng, T. Feng, and T. Zhang, "Dynamics analysis and numerical simulations of a stochastic non-autonomous predator-prey system with impulsive effects," Nonlinear Analysis: Hybrid Systems, vol. 26, pp. 19-37, 2017.

[34] S. Ruan and D. Xiao, "Global analysis in a predator-prey system with nonmonotonic functional response," SIAM Journal on Applied Mathematics, vol. 61, no. 4, pp. 1445-1472, 2001.

[35] W. Sokol and J. Howell, "Kinetics of phenol oxidation by washed cells," Biotechnology and Bioengineering, vol. 23, no. 9, pp. 20392049, 1981. 


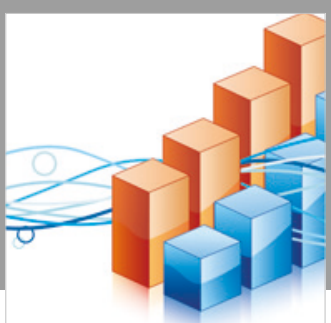

Advances in

Operations Research

\section{-n-m}
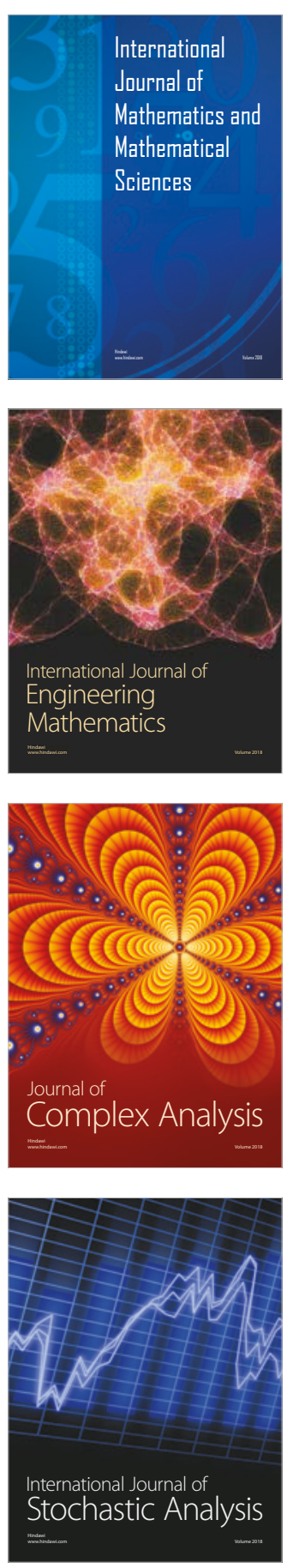
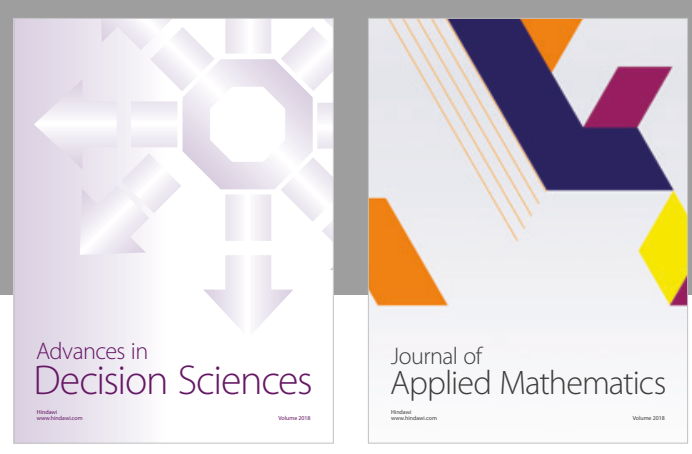

Journal of

Applied Mathematics
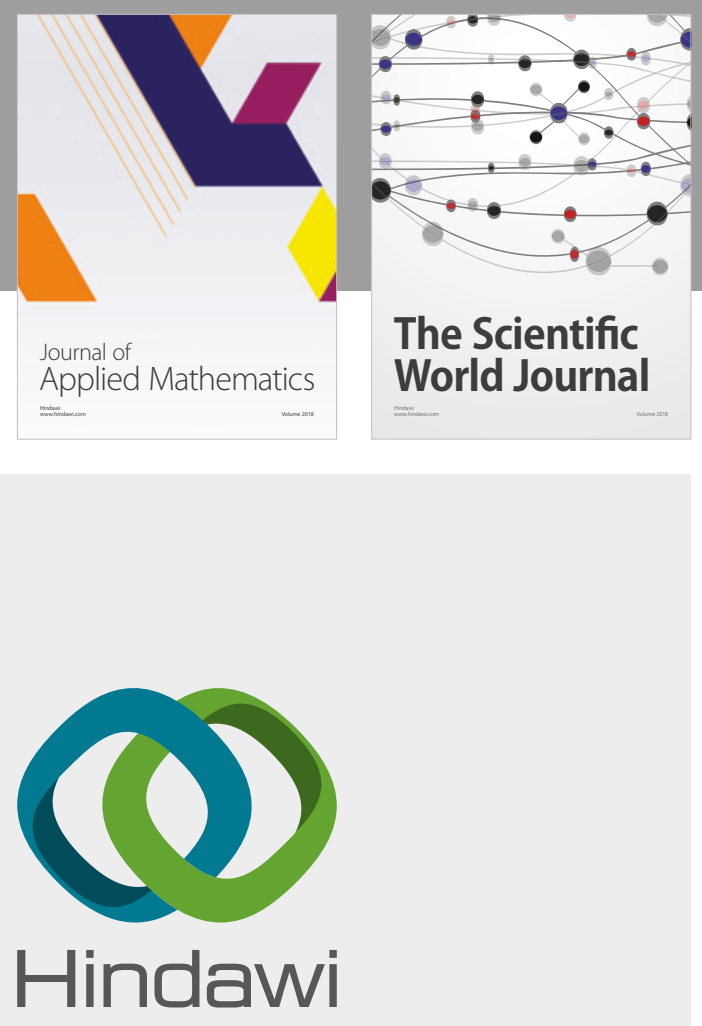

Submit your manuscripts at

www.hindawi.com

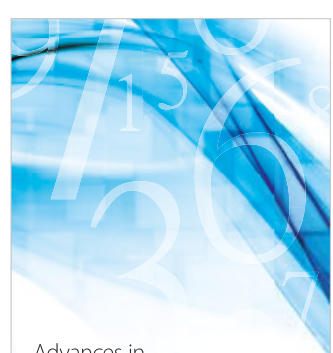

Advances in
Numerical Analysis
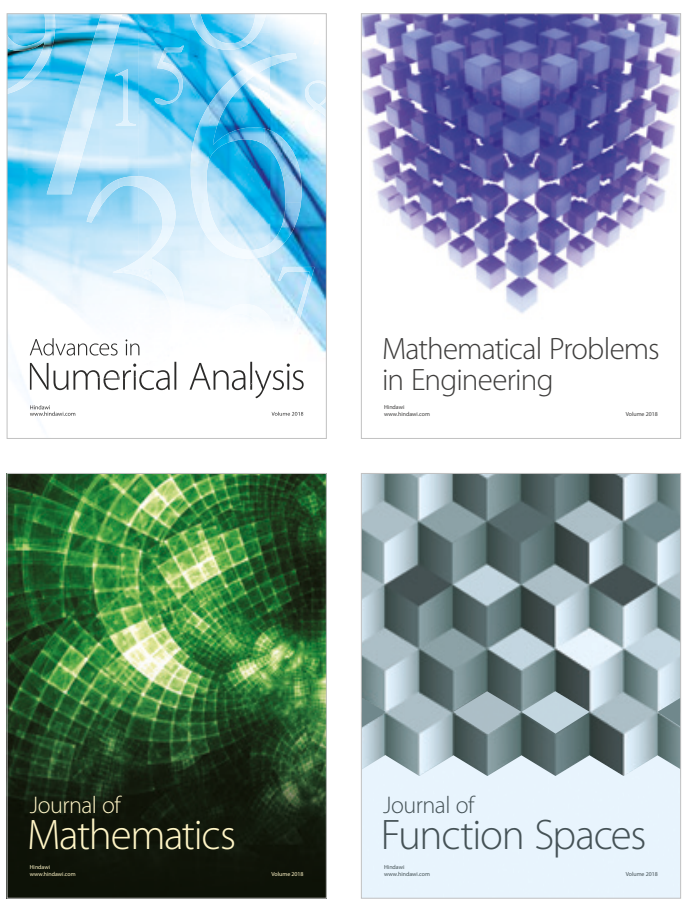

Mathematical Problems in Engineering

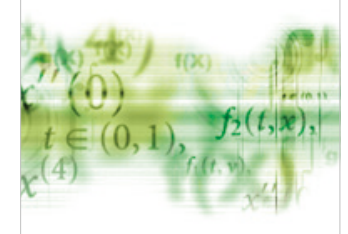

International Journal of

Differential Equations

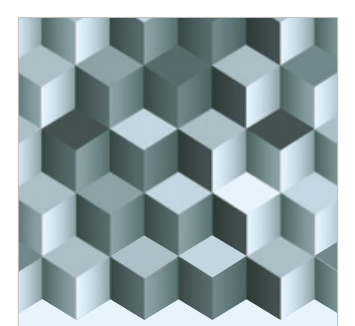

Journal of

Function Spaces
The Scientific

World Journal

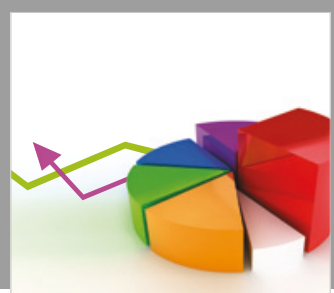

Journal of

Probability and Statistics
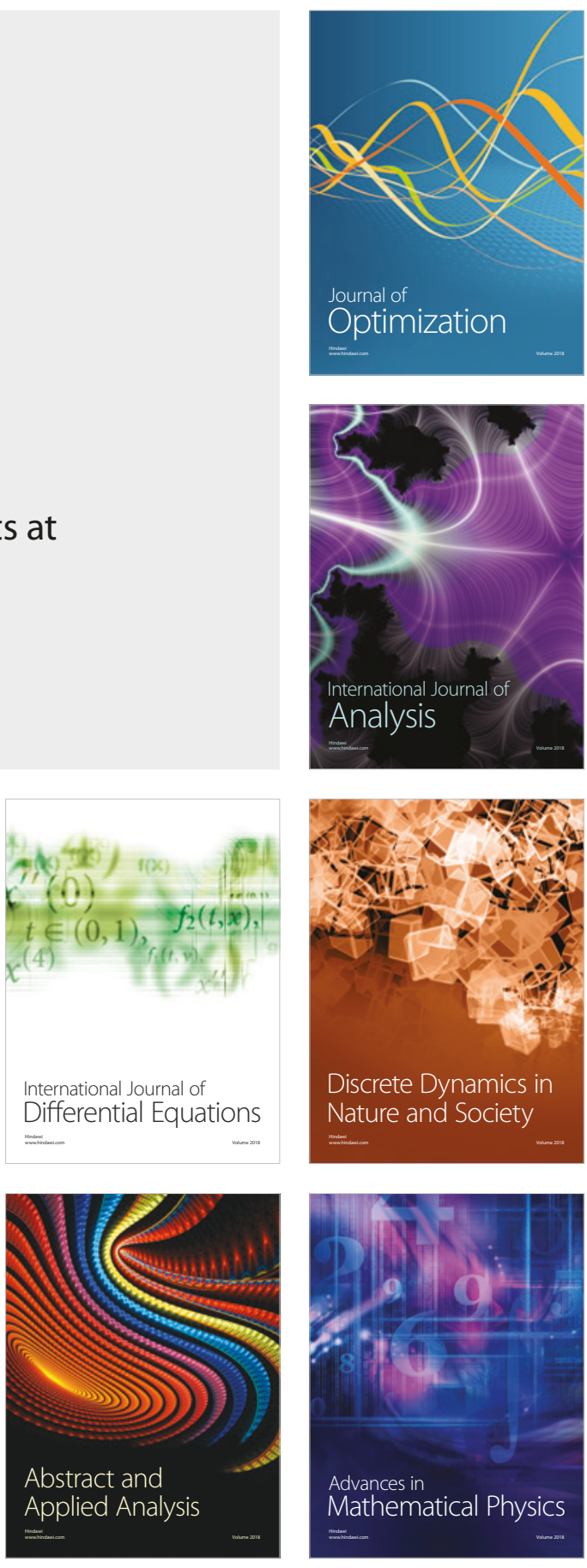